\title{
Truncated RUNX1 Generated by the Fusion of RUNX1 to Antisense GRIK2 via a Cryptic Chromosome Translocation Enhances Sensitivity to Granulocyte Colony-Stimulating Factor
}

\author{
Akihiro Abe ${ }^{a}$ Yukiya Yamamoto $^{a}$ Akira Katsumi $^{\text {b Hideyuki Yamamoto }}{ }^{a}$ \\ Akinao Okamoto $^{a}$ Yoko Inaguma $^{a}$ Chisako Iriyama $^{a}$ Masutaka Tokuda $^{a}$ \\ Masataka Okamoto ${ }^{a}$ Nobuhiko Emi ${ }^{a, c}$ Akihiro Tomita ${ }^{a}$ \\ a Department of Hematology, Fujita Health University, Toyoake, Japan; ${ }^{b}$ Department of Hematology, National \\ Center for Geriatrics and Gerontology, Obu, Japan; ' Department of Hematology, National Hospital Organization \\ Toyohashi Medical Center, Toyohashi, Japan
}

\section{Keywords}

Acute myeloid leukemia $\cdot$ GRIK2 $\cdot$ Missplicing $\cdot R U N X 1$.

Translocations

\begin{abstract}
Fusions of the Runt-related transcription factor 1 (RUNX1) with different partner genes have been associated with various hematological disorders. Interestingly, the C-terminally truncated form of RUNX1 and RUNX1 fusion proteins are similarly considered important contributors to leukemogenesis. Here, we describe a 59-year-old male patient who was initially diagnosed with acute myeloid leukemia, inv(16)(p13;q22)/CBFB-MYH11 (FAB classification M4Eo). He achieved complete remission and negative $C B F B-M Y H 11$ status with daunorubicin/cytarabine combination chemotherapy but relapsed 3 years later. Cytogenetic analysis of relapsed leukemia cells revealed CBFB-MYH11 negativity and complex chromosomal abnormalities without $\operatorname{inv}(16)$ (p13;q22). RNA-seq identified the glutamate receptor, ionotropic, kinase 2 (GRIK2) gene on $6 q 16$ as a novel fusion partner for $R U N X 1$ in this case. Specifically, the fusion of RUNX1 to the GRIK2 antisense strand (RUNX1-GRIK2as) generated multiple missplicing transcripts. Because extremely low lev-
\end{abstract}

els of wild-type GRIK2 were detected in leukemia cells, RUNX1-GRIK2as was thought to drive the pathogenesis associated with the RUNX1-GRIK2 fusion. The truncated RUNX1 generated from RUNX1-GRIK2as induced the expression of the granulocyte colony-stimulating factor (G-CSF) receptor on 32D myeloid leukemia cells and enhanced proliferation in response to G-CSF. In summary, the RUNX1-GRIK2as fusion emphasizes the importance of aberrantly truncated RUNX1 in leukemogenesis.

(c) 2020 S. Karger AG, Basel

The Runt-related transcription factor 1 (RUNX1), also known as acute myeloid leukemia 1 protein (AML1) or core-binding factor subunit alpha-2 (CBFA2), plays a crucial role in hematopoiesis. Consequently, genetic alterations in RUNX1 can induce various hematological diseases [Owen et al., 2008; Ichikawa et al., 2013]. The fusion of $R U N X 1-R U N X 1 T 1$, located at $\mathrm{t}(8 ; 21)(\mathrm{q} 22 ; \mathrm{q} 22)$, is the most frequently detected fusion gene in acute myeloid leukemia (AML) and is associated with disease maturation [Miyoshi et al., 1993]. RUNX1 also fuses with many other partner genes in hematological malignancies [De Braekeleer et al., 2009]. In most such fusions, RUNX1 re- karger@karger.com

www.karger.com/cgr

(C) 2020 S. Karger AG, Base

Karger!"
Akihiro Abe

Department of Hematology, Fujita Health University

98 Dengakugakubo, Toyoake

Aichi, 470-1192 (Japan)

aakihiro@ fujita-hu.ac.jp 
tains the runt homology domain (RHD) but loses the Cterminal transcription activation domain (TAD), suggesting that RUNX1 dysfunction is a common feature in leukemogenesis. This aberrant, C-terminally truncated form of RUNX1 is considered important in out-of-frame fusions and fusions to the antisense strands of partner genes [Maki et al., 2012; Cancer Genome Atlas Research Network, 2013; Giguère and Hébert, 2013]. For example, a short RUNX1a isoform that retains the RHD but lacks the TAD promoted the engraftment of hematopoietic stem cells in an irradiated mouse model and expansion of immature hematopoietic cells [Tsuzuki et al., 2007]. Similar to RUNX1 fusion proteins, the expression of a truncated RUNX1 protein in immature hematopoietic progenitors is considered as an important factor in the development of leukemia [Rodriguez-Perales et al., 2016].

Here, we present a case of therapy-related AML in a patient who harbored a novel variant RUNX1 fusion gene resulting from a cryptic rearrangement between $6 \mathrm{q} 16$ and 21q22. This fusion yielded a truncated form of RUNX1 that enhanced the sensitivity of leukemia cells to granulocyte colony-stimulating factor (G-CSF).

\section{Materials and Methods}

\section{RT-PCR Analysis}

TRIzol (Invitrogen, San Diego, CA, USA) was used to isolate total RNA from mononuclear bone marrow cells of our patient. Next, cDNA was synthesized from total RNA using the PrimeScript 1st strand cDNA Synthesis Kit (Takara, Otsu, Japan). RT-PCR amplification was then performed using the Platinum Taq DNA Polymerase High-Fidelity PCR kit (Invitrogen), oligonucleotide primers, and a 35-cycle protocol. The primers used for the PCR analysis are listed in online supplementary Table 1 (see www.karger.com/doi/10.1159/000508012 for all online suppl. material). The PCR reaction products were electrophoresed on $2 \%$ agarose gels and stained with ethidium bromide.

\section{Next-Generation Sequencing}

Total RNA was extracted from the patient's frozen relapsephase bone marrow cells using the RNeasy Mini-kit and QIAshredder spin column (both Qiagen, Hilden, Germany) according to the manufacturer's instructions. The integrity and purity of the total RNA were assessed using an Agilent Bioanalyzer (Agilent Technologies, Santa Clara, CA, USA). Subsequently, cDNA was generated using the NEBNext Ultra RNA Library Prep Kit for Illumina (New England Biolabs, Ipswich, MA, USA), fragmented, and processed for Illumina library preparation using NEBNext reagents (New England Biolabs). The libraries were subsequently submitted for Illumina HiSeq1500 sequencing (Illumina, San Diego, CA, USA) according to standard protocols. Approximately 51 million paired-end 100-nucleotide reads were analyzed and aligned against the human hg19 reference genome using CLC genome workbench v8.0 (Qiagen, Aarhus, Denmark). The fusion genes were also ana- lyzed using TopHat-Fusion [Kim and Salzberg, 2011] and deFuse [McPherson et al., 2011] software.

\section{Cell Culture}

The interleukin-3 (IL-3)-dependent murine myeloid cell line, $32 \mathrm{D}$, was maintained in Iscove's modified Dulbecco's medium (IMDM; Sigma, St. Louis, MO, USA) supplemented with 10\% FBS (Gibco, Grand Island, NY, USA) and $1 \mathrm{ng} / \mathrm{mL}$ murine IL-3 (mIL3; Kirin, Tokyo, Japan). G-CSF was purchased from R\&D systems (Minneapolis, MN, USA).

\section{DNA Constructs and Retroviral Vector}

RUNX1-GRIK2as cDNA of variant d (Fig. 2B) was cloned from RNA isolated from the patient's leukemia cells via RT-PCR. The amplified DNA construct was inserted into the retroviral expression vector $\mathrm{pHNGAP}$, which contains a neomycin resistance gene and a 5'-FLAG-tag [Abe et al., 2004]. This retroviral vector was subjected to nucleotide sequencing to verify the identity of the DNA.

Retrovirus particles were produced and used for transfection as described previously [Abe et al., 2004]. Briefly, the retrovirus vector plasmid and a packaging plasmid (pCGCGP) were co-transfected into 293 cells grown in 6-well plates via the calcium phosphate coprecipitation method. The pseudotyped viruses were collected $48 \mathrm{~h}$ after transfection and stored at $-80^{\circ} \mathrm{C}$ until further use.

These retroviral vectors were used to infect $32 \mathrm{D}$ cells in the presence of $10 \mu \mathrm{g} / \mathrm{mL}$ protamine (Mochida Pharmaceutical Co. Ltd, Tokyo, Japan). G418 (GIBCO) was added after $48 \mathrm{~h}$ to select stable transfectants and establish $32 \mathrm{D}$ cell lines expressing truncated RUNX1 (32D/RUNX1_TR). The expression of the Flagtagged protein from transfected vectors was confirmed by Western blotting with the M2 antibody (Sigma).

\section{Flow Cytometry}

The 32D and 32D/RUNX1_TR cells were incubated with phycoerythrin-conjugated anti-mouse Gr1 (eBioscience, San Diego, CA, USA) or FITC-conjugated anti-mouse G-CSF receptor (GCSFR; Becton Dickinson, San Jose, CA, USA) for 30 min on ice and then washed with phosphate-buffered saline. The flow cytometric analysis was performed using a BD FACSCanto II flow cytometer and FACSDiva software (Becton Dickinson).

\section{Cell Proliferation (XTT) Assay}

The cells were seeded into 96-well plates at a density of $10^{4} /$ well in $100 \mu \mathrm{L}$ IMDM supplemented with $10 \%$ horse serum (GIBCO), $0.01 \mathrm{ng} / \mathrm{mL} \mathrm{mIL}-3$, and various concentrations of G-CSF. The plates were incubated at $37^{\circ} \mathrm{C}$ for $72 \mathrm{~h}$ before addition of sodium $3^{\prime}$-[1-(phenylaminocarbonyl)-3,4-tetrazolium]-bis(4-methoxy6-nitro)benzene sulfonic acid hydrate (XTT; Dojin Chemical, Kumamoto, Japan). After $2 \mathrm{~h}$ incubation, the absorption in each well at $450 \mathrm{~nm}$ was measured using a microplate reader.

\section{Results}

\section{Patient}

The 59-year-old man was initially diagnosed with AML with eosinophilia (FAB classification M4Eo) (Fig. 1B) and admitted to Fujita Health University in Au- 
Fig. 1. Clinical course and morphological analysis of leukemia cells. A Chemotherapy regimen, $C B F B-M Y H 11$, and disease state are shown. IA, idarubicin + Ara-C; MA, mitoxantrone + Ara-C; DA, daunorubicin + Ara-C; AA, aclarubicin + Ara-C; CAG, Ara-C + aclarubicin + G-CSF; CR, complete remission. B At initial diagnosis, bone marrow aspirate smears revealed myelomonocytic blasts with eosinophils. C At secondary AML, bone marrow aspirate smears revealed monoblastic leukemia cells with vacuoles. B, C May-GrünwaldGiemsa staining, $\times 400$.

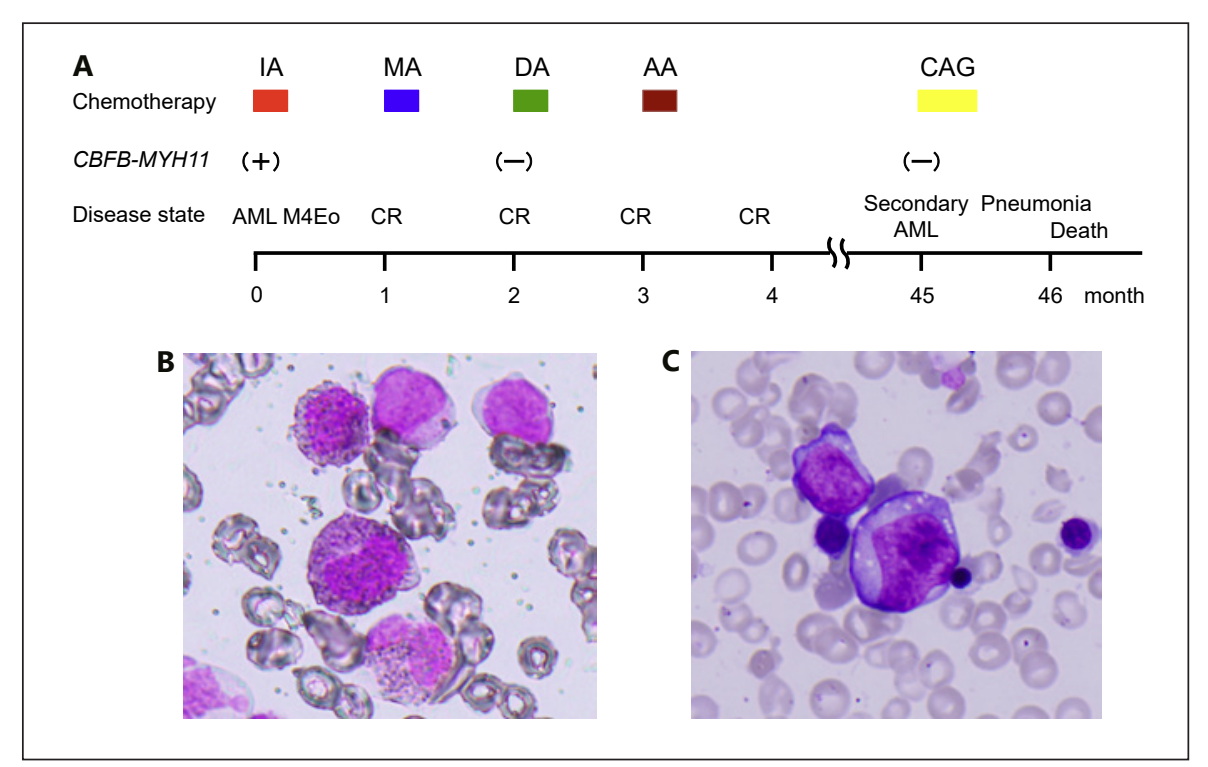

gust 2008. His leukemia blasts exhibited the karyotype $46, \mathrm{XY}, \operatorname{inv}(16)(\mathrm{p} 13 \mathrm{q} 22)$ and were positive for the corebinding factor subunit beta-myosin-heavy chain 11 (CBFB-MYH11) fusion gene. He was treated with a chemotherapy regimen of IA (idarubicin, cytarabine [AraC]), followed by 3 courses of consolidation therapy: MA (mitoxantrone, Ara-C), DA (daunorubicin, Ara-C), and AA (aclarubicin, Ara-C) (Fig. 1A). He achieved complete remission. However, he suffered a leukemia relapse 3 years later.

A bone marrow aspiration at the time of relapse revealed predominant monoblastic leukemia cells and an increased population of immature erythroid cells exhibiting dysplastic morphology (Fig. 1C). The relapsed leukemia blasts exhibited complex chromosomal aberrations without inv(16)(p13q22), and RT-PCR analysis did not detect the CBFB-MYH11 fusion. A cytogenetic analysis of the bone marrow cells yielded the following karyotype: $44, \mathrm{XY},-3, \operatorname{der}(5) \mathrm{t}(5 ; 17)$ (q11.2;q11.2)ins(5;?)(q11.2;?),-6, $-14,-17,+\operatorname{mar} 1,+\operatorname{mar} 2[1] / 43$, idem, $-7,-18,-21,+\operatorname{der}(?)$ $\mathrm{t}(? ; 7)(? ; \mathrm{q} 11.2),+\operatorname{mar} 3[4] / 44, \mathrm{idem}, \operatorname{der}(7 ; 14)(\mathrm{q} 10 ; \mathrm{q} 10),-21$, + mar3,+mar4[2]. Upon analysis of single nucleotide variants (SNVs) from RNA-seq data, we found that the alteration of p53 G245S occurred at relapse with an allele frequency of $93.5 \%$ (online suppl. Table 2). Furthermore, we discovered that NOTCH1 Q1134R and other SNVs with high pathogenic scores were common in both initial and relapsed leukemia. Based on these findings, the patient was diagnosed with therapy-related AML. A single course of salvage therapy with CAG (Ara-C, aclarubicin,
G-CSF) did not improve the patient's condition. He finally died of pneumonia and leukemia progression.

Identification of RUNX1-GRIK2as Fusion Transcripts

As described in Materials and Methods, we obtained and analyzed more than 51 million short paired-end reads from the patient's leukemia cDNA library. An RNA-seq analysis revealed a novel fusion of RUNX1 at $21 \mathrm{q} 22$ with the antisense strand of the gene encoding glutamate receptor, ionotropic, kinase 2 (GRIK2) at 6q16 (RUNX1-GRIK2as; Fig. 2A). Fluorescence in situ hybridization (FISH) with a dual-color dual fusion translocation probe directed at the RUNX1/RUNX1T1 chimera (Abbott Molecular/Vysis, Des Plaines, IL, USA) suggested the splitting of RUNX1 signals (Fig. 2B). However, the cells were negative for the RUNX1-RUNX1T1 fusion, and a spectral karyotyping analysis (SRL, Tokyo, Japan) did not reveal a rearrangement of $6 \mathrm{q} 16$ and $21 \mathrm{q} 22$ (online suppl. Fig. 1). In other words, the rearrangement between 6q16 and 21q22 was a cryptic abnormality.

\section{Multiple RUNX1-GRIK2as Transcripts and PCR Analysis}

We identified 5 fusion sites in GRIK2: 3 sites at intron 13 and 2 sites at intron 14 (Fig. 2A, 3A). Exon 6 of RUNX1 was dominantly fused to the reverse sequence of GRIK2, whereas alternative splicing transcripts of RUNX1 exon 5 were fused to GRIK2 in each fusion transcript (Fig. 3A). A fusion to an antisense sequence may disrupt the normal splicing machinery via arbitrary splicing acceptors and 


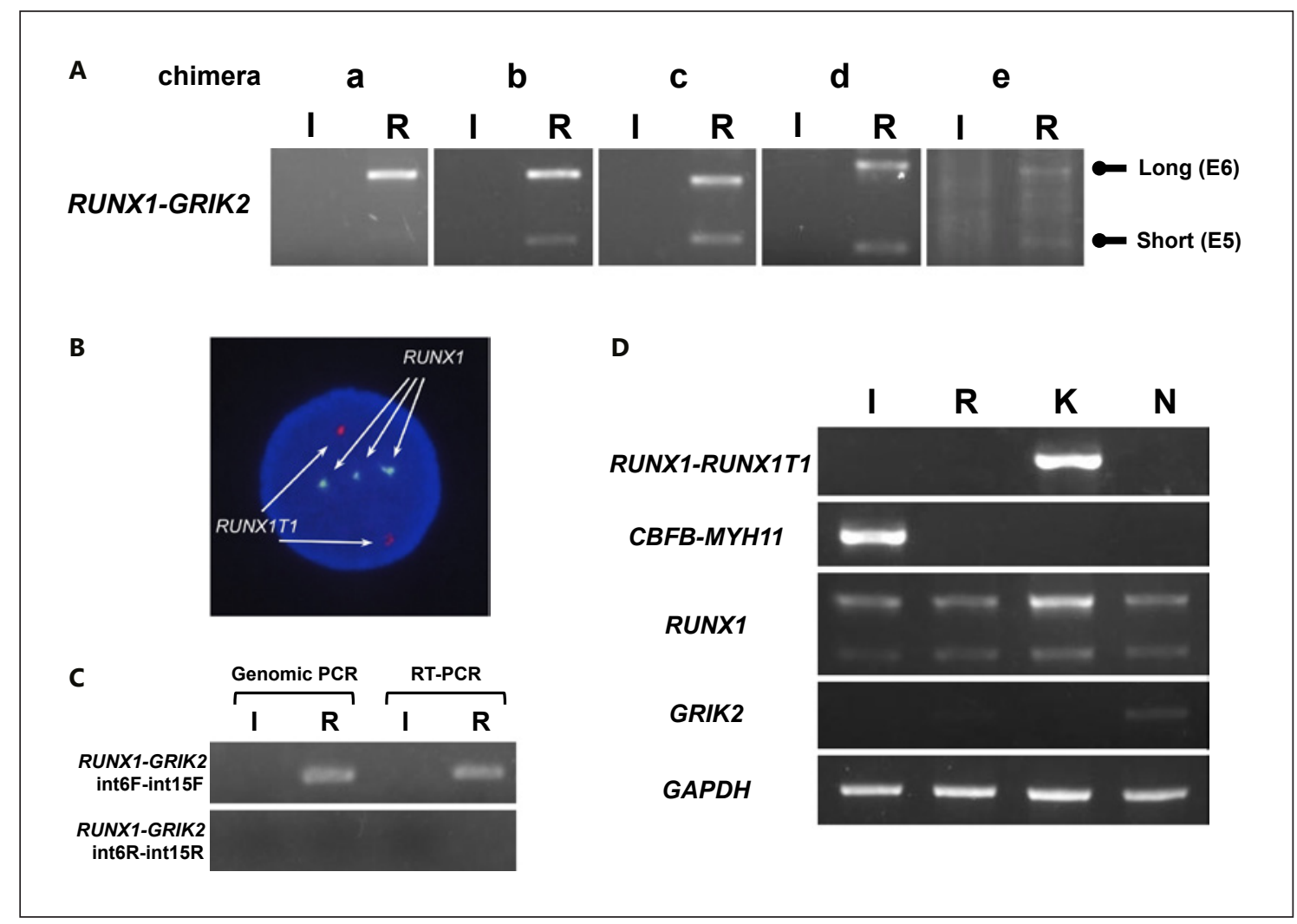

Fig. 2. PCR and FISH analyses. A RT-PCR analysis of RUNX1GRIK2 fusion genes. The fusion variants with splicing at the donor sites corresponding to the exon 5 -intron 5 or exon 6 -intron 6 junctions of RUNX1 are defined as the short (E5) and long (E6) forms, respectively. B FISH analysis with a dual-color dual fusion translocation probe for the RUNX1/RUNX1T1 chimeric gene yielded 3 RUNX1 signals (blue), suggesting the splitting of this gene. The RUNX1-RUNX1T1 fusion status was negative. C PCR analysis of the genomic breakpoint. The RUNX1-GRIK2as PCR product amplified from genomic DNA was the same size as that amplified

can yield multiple missplicing products. The alternative splicing transcripts used a standard splicing donor at the junctions of exon 5-intron 5 and exon 6-intron 6 of $R U N X 1$, but unusual splicing acceptors in the antisense strand of GRIK2 (Fig. 3B). The GT-AG splicing sites remained regulated in these transcripts. Approximately $58 \%$ of leukemia clones harbored RUNX1-GRIK2as according to an estimate from the number of reads mapped to the RUNX1 ex6-ex7 or RUNX1 ex6-GRIK2 junction (Fig. 4A). The type d fusion transcript of RUNX1 ex6GRIK2as appeared to be the most frequent (Fig. 4A). Moreover, the very low number of reads mapped to the $5^{\prime}$-region of GRIK2 suggested that the transcriptional activity of GRIK2 was repressed (Fig. 4B). from cDNA (primers: RUNX1_int6F, GRIK2_int15F). D Expression patterns of CBFB-MYH11, RUNX1, and GRIK2. CBFBMYH11 was determined at the initial diagnosis, but was negative at the time of relapse. Wild-type RUNX1 and 2 splicing transcripts were detected at both time points and in the normal bone marrow, whereas GRIK2 was detected in normal bone marrow and was absent or detectable at very low levels in leukemia cells. GAPDH was used as a positive control. I, initial diagnosis; $\mathrm{R}$, relapse; $\mathrm{K}$, the $\mathrm{t}(8$; 21) Kasumi-1 cell line; N, normal bone marrow.

Our RNA-seq analysis further identified a genomic breakpoint located in RUNX1 intron 6 and GRIK2 intron 15 (Fig. 3A). The RT-PCR amplification band between the $5^{\prime}$-ends of RUNX1 intron 6 and GRIK2 intron 15 was identical to that generated by the PCR amplification of genomic DNA using the same primers. The intronic sequences around the breakpoint were transcribed to RNA, and a reciprocal fusion of the $3^{\prime}$-end of RUNX1 intron 6 to the $3^{\prime}$-end of GRIK2 intron 15 was not detected (Fig. 2C). No expression of GRIK2 was observed in the patient's relapsed leukemia cells (Fig. 2D), which expressed the normal allele of $R U N X 1$ (Fig. 2D) with no detectable mutation. 


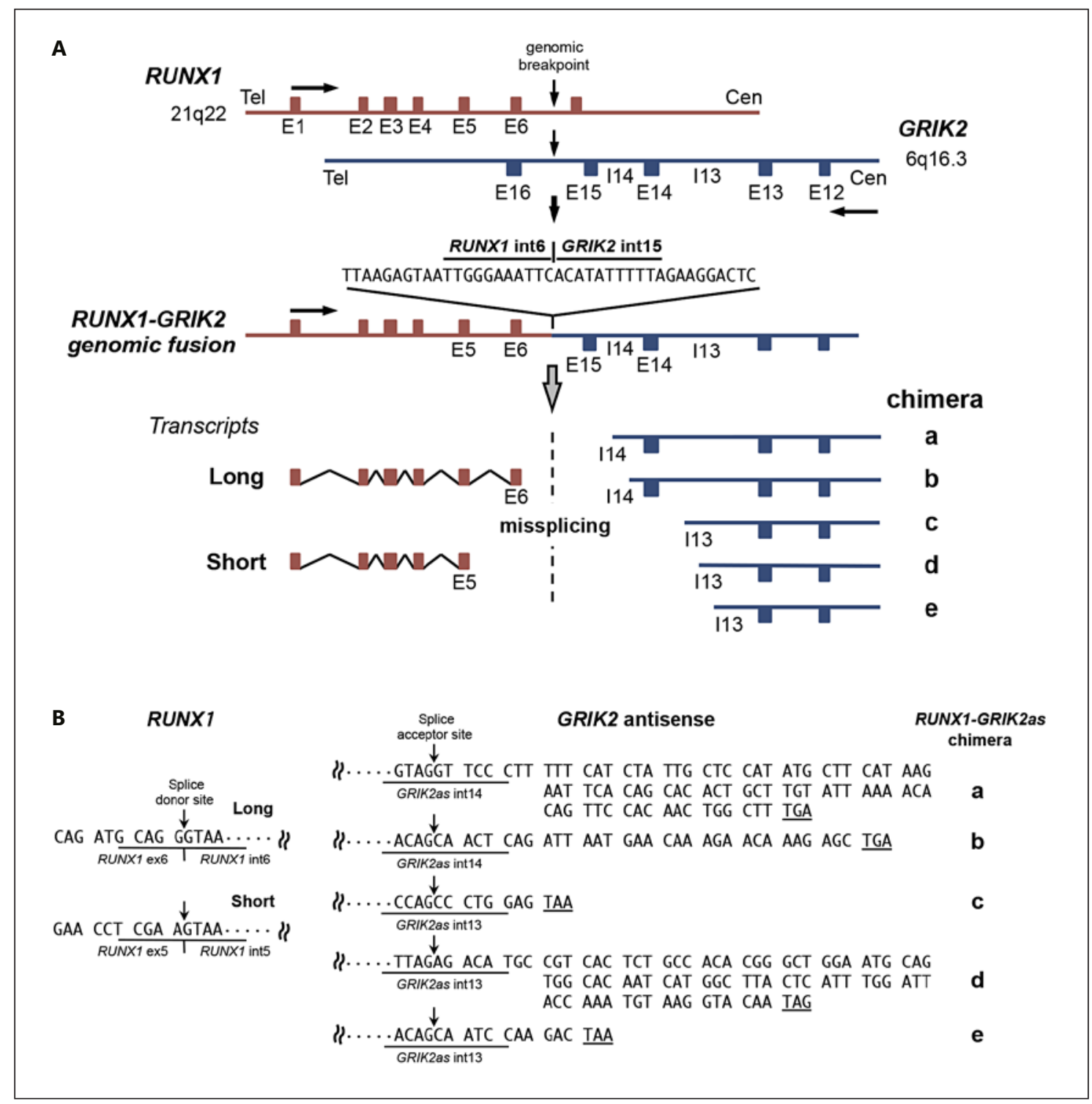

Fig. 3. Schematic structure and sequence of the RUNX1-GRIK2as fusion. A The fusion transcripts were transcribed by the RUNX1 promoter and fused to the antisense strands of GRIK2 (RUNX1GRIK2as). RUNX1 was spliced at the exon 5 -intron 5 (short) or exon 6-intron 6 (long) junction to form the RUNX1-GRIK2as fusion. The antisense strand of GRIK2 contained 5 splicing acceptor sites, and fusions with these different sites are designated as chimeras a-e. B DNA sequences around the fusion sites. The reading frame is distinguished by a space. Predicted stop codons are underlined. The common sequences of the splicing donor sites (GT) and acceptor sites (AG) were conserved. E, exon; I, intron; Tel, telomere; Cen, centromere.
Enhanced Proliferation of 32D/RUNX1_TR Cells in Response to G-CSF

The 32D cells transduced stably with the vector encoding RUNX1-GRIK2as expressed truncated RUNX1 but could not be maintained without mIL-3. Moreover, these cells did not exhibit any proliferative advantage in response to mIL-3. We next analyzed the proliferative re- sponse of these 32D/RUNX1_TR cells to G-CSF, as the expression of G-CSFR on the surfaces of 32D cells is induced by mIL-3 depletion. As G-CSF was not sufficient for the maintenance of these cells, we examined the proliferative response to G-CSF in the presence of a low concentration of mIL-3 $(0.01 \mathrm{ng} / \mathrm{mL})$. Notably, the 32D/ RUNX1_TR cells exhibited an enhanced proliferative re- 
Fig. 4. Analysis of RUNX1 and GRIK2 mapping data. A The numbers of reads mapped to the RUNX1 ex6-ex7 junction and RUNX1 ex6-GRIK2 junction. The reads mapped to the RUNX1 ex6-GRIK2 junction were subclassified to variants labeled chimeras a-e. Variant chimera d was dominant. The estimated percentage of leukemia clones harboring RUNX1GRIK2as was approximately 58\%. B The number of reads mapped to the GRIK2 exon and intron. The $5^{\prime}$-region of GRIK2 was not expressed. Therefore, the antisense strand of the $3^{\prime}$-region of GRIK2 was transcribed from RUNX1 in RUNX1-GRIK2as.
A

\begin{tabular}{|c|c|c|c|c|c|c|}
\hline & $\begin{array}{c}\text { Reads mapped on } \\
\text { RUNX1 ex6-ex7 junction }\end{array}$ & \multicolumn{5}{c|}{$\begin{array}{c}\text { Reads mapped on } \\
\text { RUNX1 ex6-GRIK2 junction }\end{array}$} \\
\hline Number of reads & 95 & \multicolumn{5}{|c|}{39} \\
\hline Variants & - & $\mathrm{a}$ & $\mathrm{b}$ & $\mathrm{c}$ & $\mathrm{d}$ & $\mathrm{e}$ \\
\hline Number of reads & - & 2 & 2 & 2 & 27 & 6 \\
\hline
\end{tabular}

\begin{tabular}{|c|c|c|c|c|c|c|c|c|c|c|c|c|c|c|c|c|}
\hline & \multicolumn{11}{|c|}{ GRIK2 } \\
\hline Exon no. & 1 & 2 & 3 & 4 & 5 & 6 & 7 & 8 & 9 & 10 & 11 & 12 & 13 & 14 & 15 & 16 \\
\hline Number of reads & 1 & 0 & 0 & 0 & 0 & 0 & 0 & 0 & 0 & 0 & 2 & 0 & 0 & 7 & 2 & 1 \\
\hline Intron no. & 1 & 2 & 3 & 4 & 5 & 6 & 7 & 8 & 9 & 10 & 11 & 12 & 13 & 14 & 15 & - \\
\hline Number of reads & 2 & 0 & 0 & 0 & 14 & 21 & 0 & 2 & 22 & 33 & 26 & 1 & $>300$ & 97 & 3 & - \\
\hline
\end{tabular}

A

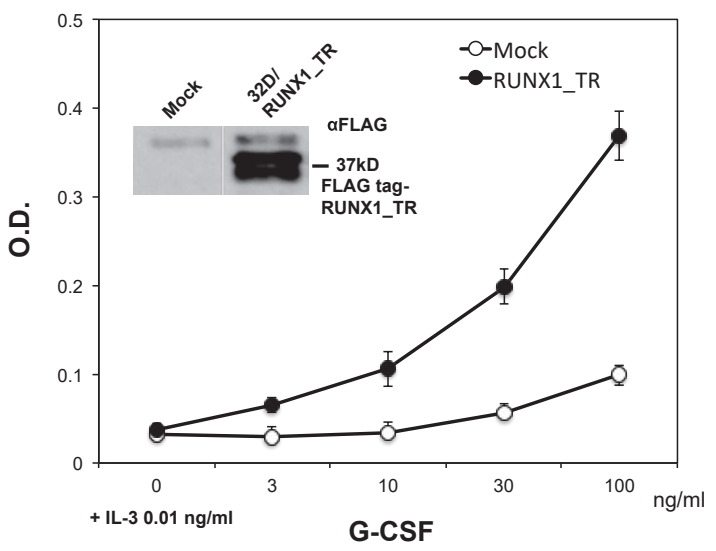

B

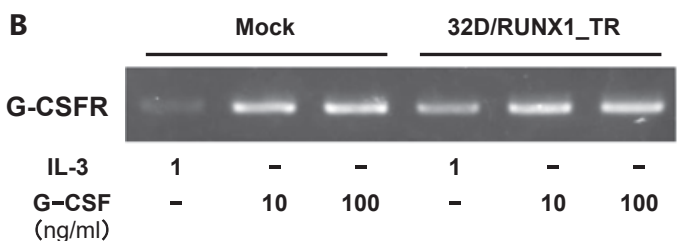

C

Mock
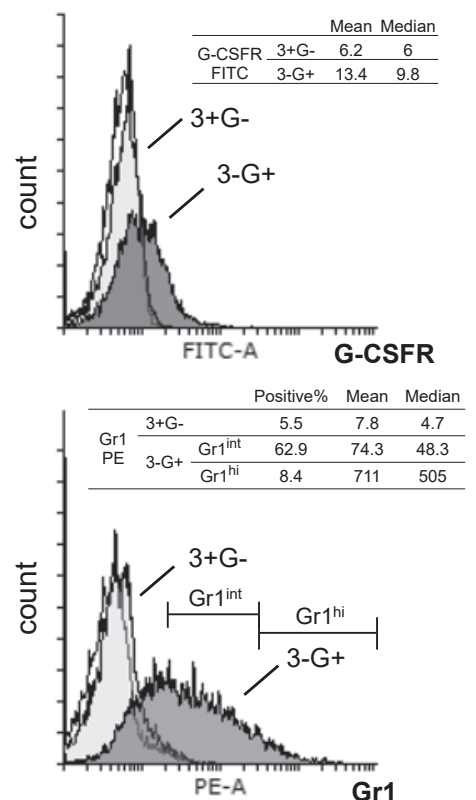

32D/RUNX1_TR
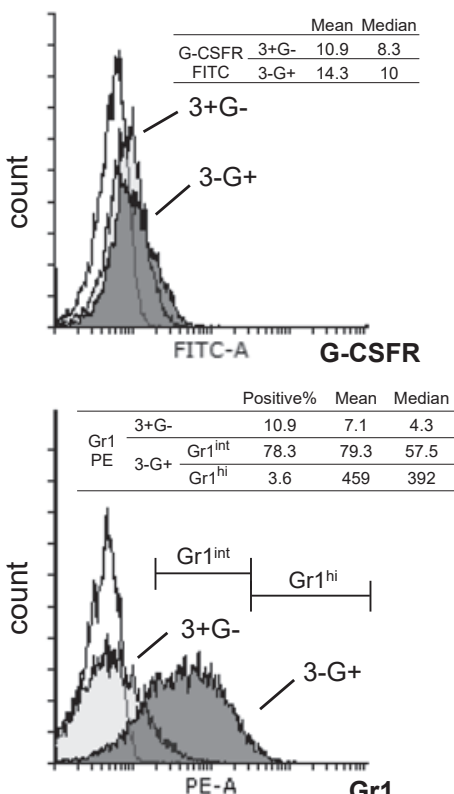

Fig. 5. Proliferation and differentiation of 32D/RUNX1_TR cells in response to G-CSF. A Proliferative responses of 32D/Mock and 32D/RUNX1_TR cells to G-CSF. The latter cells were infected with a lentiviral vector engineered to express truncated RUNX1. The cells $\left(10^{4}\right.$ per well) were cultured for 3 days in the presence of 0.01 $\mathrm{ng} / \mathrm{mL} \mathrm{mIL}-3$ and the indicated concentrations of G-CSF. Viable cells were measured using the XTT assay. A Western blot analysis demonstrates the presence of truncated RUNX1 protein $(37 \mathrm{kDa})$ in 32D/RUNX1_TR cells. The data are shown from one representative of 3 replicated independent experiments. B RT-PCR analysis of the expression of the G-CSF receptor gene (GCSFR) in 32D/
Mock and 32D/RUNX1_TR cells. The expression of GCSFR was induced after depleting mIL-3 and adding G-CSF. The expression of GCSFR in 32D/RUNX1_TR cells was higher than that in wildtype $32 \mathrm{D}$ cells cultured in the presence of mIL-3. C Flow cytometry analysis of the expression of G-CSFR and the myeloid differentiation antigen Gr1 in 32D/Mock and 32D/RUNX1_TR cells cultured in the presence of $100 \mathrm{ng} / \mathrm{mL}$ G-CSF for 5 days. Both cell types were cultured in IMDM supplemented with $10 \%$ FBS. G-CSFR was expressed on 32D/RUNX1_TR cells even in the presence of mIL-3. Representative data are shown. 
Table 1. C-terminally truncated RUNX1 resulting from RUNX1 translocation

\begin{tabular}{|c|c|c|c|c|c|c|}
\hline $\begin{array}{l}\text { Original name of } \\
\text { chimeric gene }\end{array}$ & $\begin{array}{l}\text { HGNC symbol } \\
\text { of partner gene }\end{array}$ & Location & Type of disease & \multicolumn{2}{|c|}{ Breakpoints of fusion transcripts } & Reference \\
\hline RUNX1-EAP & RPL22 & $3 q 26$ & $\begin{array}{l}\text { tAML, tMDS, CML } \\
\text { BC }\end{array}$ & $\begin{array}{l}\text { exon } 5 \\
\text { exon } 6\end{array}$ & RPL22: exon 2, out of frame & Nucifora et al., 1994 \\
\hline RUNX1-AMP19 & $\mathrm{ND}$ & $19 q 13.4$ & $\begin{array}{l}\text { Radiation-associated } \\
\text { AML }\end{array}$ & $\begin{array}{l}\text { exon } 5 \\
\text { exon } 6\end{array}$ & $\begin{array}{l}\mathrm{IGR}^{\mathrm{a}} \\
\text { to NLRP4: intron } 8 \text {, antisense }\end{array}$ & Hromas et al., 2001 \\
\hline RUNX1-Copine VIII & CPNE8 & $12 \mathrm{q} 12$ & AML & exon 6 & $\mathrm{IGR}^{\mathrm{b}}$ & Ramsey et al., 2003 \\
\hline RUNX1-SV2B & $S V 2 B$ & $15 q 26.1$ & AML & exon 1 & $S V 2 B$ : intron 1 , antisense & Giguère and Hébert, 2013 \\
\hline RUNX1-TMEM48 & $N D C 1$ & $1 \mathrm{p} 32$ & MDS/AML & intron 5 & NDC1: intron 3 , antisense & Rodriguez-Perales et al., 2016 \\
\hline ND & ND & $6 \mathrm{q} 25$ & AML & exon 6 & IGR & Panagopoulos et al., 2016 \\
\hline RUNX1-PRPF38A & PRPF38A & $1 \mathrm{p} 32$ & AML & exon 6 & PRPF38A: exon 1, out of frame & Wang et al., 2017 \\
\hline RUNX1-GRIK2 & GRIK2 & $6 \mathrm{q} 16.3$ & AML relapse & $\begin{array}{l}\text { exon } 5 \\
\text { exon } 6\end{array}$ & $\begin{array}{l}\text { GRIK2: intron } 13 \text {, antisense } \\
\text { intron } 14 \text {, antisense }\end{array}$ & Present case \\
\hline
\end{tabular}

CML BC, chronic myeloid leukemia blast crisis; HGNC, HUGO Gene Nomenclature Committee; IGR, intergenic region; ND, not determined; tAML, therapy-related acute myeloid leukemia; tMDS, therapy-related myelodysplasia. ${ }^{a}$ Between NLRP4 and NLRP13. ${ }^{\mathrm{b}} 73 \mathrm{~kb}$ upstream of CPNE8.

sponse to G-CSF (Fig. 5A). Interestingly, RUNX1_TR induced the expression of G-CSFR even in the presence of mIL-3 (Fig. 5B, C).

Grl is a cell surface antigen expressed during the maturation of myeloid cells. G-CSF induces the granulocytic differentiation of 32D cells by upregulating the expression of Gr1 [Schuster et al., 2003]. Accordingly, we assessed the frequencies of $\mathrm{Gr} 1^{\text {int }}$ and $\mathrm{Gr} 1^{\text {hi }}$ cells to determine the effect of G-CSF on the myeloid differentiation of $32 \mathrm{D}$ cells. Although RUNX1_TR did not impair the expression of $\mathrm{Gr} 1$ on 32D cells in the presence of G-CSF, a mild increase in the $\mathrm{Gr} 1{ }^{\mathrm{int}}$ fraction and modest decrease in the $\mathrm{Gr} 1^{\text {hi }}$ fraction were observed in 32D/RUNX1_TR cells relative to $32 \mathrm{D}$ cells (Fig. 5C).

\section{Discussion}

In this case, our patient was initially diagnosed with AML harboring the CBFB-MYH11 fusion. Upon relapse, however, his leukemia cells were negative for $C B F B$ MYH11 and exhibited complex chromosomal abnormalities. An RNA-seq analysis revealed the occasional fusion of RUNX1 at $21 \mathrm{q} 22$ to GRIK2 at $6 \mathrm{q} 16$ via a cryptic rearrangement that was not identified by G-band and spectral karyotyping analyses.

TP53 mutations have an increased incidence rate in therapy-related AML [Wong et al., 2015]. Initial leukemia cells were diagnosed as AML M4Eo with several pathogenic SNVs in addition to CBFB-MYH11, in particular NOTCH1 Q1134R, which is known as a recurrent alteration in Chinese oral squamous cell carcinoma [Song et al., 2014]. Although CBFB-MYH11 was not detected in the relapsed leukemia cells, based on the preleukemic clones harboring pathogenic SNVs, we believe that the TP53 mutation caused therapy-related AML.

Glutamate receptors mediate the majority of excitatory neurotransmissions in the brain. GRIK2 produces a protein with a distinct role in glutamatergic neurotransmission and neuronal development as well as a potential tumor-suppressing capacity [Sinclair et al., 2004]. Notably, TRMT11-GRIK2 fusion genes have been reported in $7.9 \%$ of prostate cancer cases, where the loss of function is predicted to contribute to tumorigenesis [ $\mathrm{Yu}$ et al., 2014]. In our case of AML, very low levels of expression of the $5^{\prime}$-end of GRIK2 were detected in the patient's leukemia cells via RNA-seq and RT-PCR analyses. Therefore, the pathogenesis of the RUNX1-GRIK2 fusion may depend on the aberrantly truncated RUNX1 product produced by RUNX1-GRIK2as.

To date, 9 types of translocations predicted to produce aberrantly truncated forms of RUNX1 have been reported (Table 1). RUNX1-EAP is an out-of-frame fusion [Nucifora et al., 1994], while RUNX1-AMP19 and RUNX1Copine VIII [Hromas et al., 2001; Ramsey et al., 2003] are fusions of RUNX1 to noncoding intergenic regions. Panagopoulos et al. [2016] reported a similar RUNX1 truncation resulting from a fusion to an intergenic sequence 
on 6q25. RUNX1-DTX2, RUNX1-SV2B, and RUNX1TMEM48 are fusions with the antisense strands of the respective partner genes. These fusions disrupt the normal splicing machinery and yield fusion transcripts that include intron regions [Maki et al., 2012; Giguère and Hébert, 2013; Rodriguez-Perales et al., 2016]. RUNX1$P R P F 38 A$ is a fusion of RUNX1 to the $5^{\prime}$ noncoding sequence in exon 1 of PRPF38A [Wang et al., 2017]. Most fusion sites of RUNX1 involve exon 6, although RUNX1$S V 2 B$ and RUNX1-TMEM48 involve exon 1 and intron 5, respectively. RUNX1-DTX2 produces an aberrant C-terminally truncated RUNX1 via the fusion of RUNX1 to $U P K 3 B-D T X 2$ reverse sequences. This truncated protein is reported to act as a dominant negative inhibitor of RUNX1 [Maki et al., 2012].

The exogenous expression of truncated RUNX1 from RUNX1-TMEM48 increases the proliferation and self-renewal of human hematopoietic/progenitor stem cells [Rodriguez-Perales et al., 2016]. Chin et al. [2016] reported that in mouse bone marrow, Runx 1 haploinsufficiency promotes the hypersensitivity of hematopoietic stem and progenitor cells to G-CSF and impedes G-CSF-induced granulopoiesis, leading to the accumulation of $\mathrm{Gr} 1^{\text {int }}$ granulocytes. Here, we demonstrate that in $32 \mathrm{D}$ cells, truncated RUNX1 enhanced proliferation in response to G-CSF and induced G-CSFR expression. In these cells, RUNX1_TR expression led to a mild increase of the Gr $1^{\text {int }}$ fraction in the presence of G-CSF.

Taken together, we have identified GRIK2 as a novel fusion partner for RUNX1, and demonstrated that this fusion is chimerized in the reverse direction via a cryptic chromosome translocation between $6 \mathrm{q} 16$ and $21 \mathrm{q} 22$. This RUNX1-GRIK2as fusion supports the importance of aberrantly truncated RUNX1 in leukemogenesis.

\section{Acknowledgements}

We greatly appreciate the technical assistance of Dr. Hidehito Inagaki and Dr. Hiroki Kurahashi (Genome and Transcriptome Analysis Center, FHU) during next-generation sequencing. We also thank Sachiko Iba and Keiko Hattori for their valuable laboratory assistance. The authors would like to thank Enago (www.enago.jp) for the English language review.

\section{Statement of Ethics}

The patient provided written informed consent for the isolation of mononuclear cells from his bone marrow. This study has been approved by Human Genome, Gene Analysis Research Ethics Committee of Fujita Health University.

\section{Disclosure Statement}

The authors have no conflicts of interest relevant to this report to declare.

\section{Funding Sources}

This work was supported by JSPS KAKENHI Grant Number 16K09860 to A.A., a Grant-in-Aid from Fujita Health University to N.E., and the Program for the Strategic Research Foundation at Private Universities supported by the Ministry of Education, Culture, Sports, Science, and Technology (MEXT).

\section{Author Contributions}

A.A. and Y.Y. performed the research and the gene analysis, and drafted the manuscript. N.E. and A.T. designed the research. A.K. revised the manuscript and added various comments as well as the interpretation of the results. H.Y., A.O., Y.I., C.I., M.T., and M.O. assessed the clinical data of the patient. All authors read and approved the final manuscript.

\section{References}

Abe A, Emi N, Kanie T, Imagama S, Kuno Y, et al: Expression cloning of oligomerization-activated genes with cell-proliferating potency by pseudotype retrovirus vector. Biochem Biophys Res Commun 320:920-926 (2004).

Cancer Genome Atlas Research Network: Genomic and epigenomic landscapes of adult de novo acute myeloid leukemia. $\mathrm{N}$ Engl J Med 368:2059-2074 (2013).

Chin DW, Sakurai M, Nah GS, Du L, Jacob B, et al: RUNX1 haploinsufficiency results in granulocyte colony-stimulating factor hypersensitivity. Blood Cancer J 6:e379 (2016).
De Braekeleer E, Ferec C, De Braekeleer M: RUNX1 translocations in malignant hemopathies. Anticancer Res 29:1031-1037 (2009).

Giguère A, Hébert J: Identification of a novel fusion gene involving $R U N X 1$ and the antisense strand of $S V 2 B$ in a $B C R-A B L 1$-positive acute leukemia. Genes Chromosomes Cancer 52: 1114-1122 (2013).

Hromas R, Busse T, Carroll A, Mack D, Shopnick R, et al: Fusion AML1 transcript in a radiation-associated leukemia results in a truncated inhibitory AML1 protein. Blood 97:2168-2170 (2001).
Ichikawa M, Yoshimi A, Nakagawa M, Nishimoto N, Watanabe-Okochi N, Kurokawa M: A role for RUNX1 in hematopoiesis and myeloid leukemia. Int J Hematol 97:726-734 (2013).

Kim D, Salzberg SL: TopHat-Fusion: an algorithm for discovery of novel fusion transcripts. Genome Biol 12:R72 (2011).

Maki K, Sasaki K, Sugita F, Nakamura Y, Mitani $\mathrm{K}$ : Acute myeloid leukemia with $\mathrm{t}(7 ; 21)$ (q11.2;q22) expresses a novel, reversed-sequence RUNX1-DTX2 chimera. Int J Hematol 96:268-273 (2012). 
McPherson A, Hormozdiari F, Zayed A, Giuliany R, Ha G, et al: deFuse: an algorithm for gene fusion discovery in tumor RNASeq data. PLoS Comput Biol 7:e1001138 (2011).

Miyoshi H, Kozu T, Shimizu K, Enomoto K, Maseki N, et al: The $t(8 ; 21)$ translocation in acute myeloid leukemia results in production of an AML1-MTG8 fusion transcript. EMBO J 12:2715-2721 (1993).

Nucifora G, Begy CR, Kobayashi H, Roulston D, Claxton D, et al: Consistent intergenic splicing and production of multiple transcripts between $A M L 1$ at $21 \mathrm{q} 22$ and unrelated genes at $3 \mathrm{q} 26$ in $(3 ; 21)$ (q26;q22) translocations. Proc Natl Acad Sci USA 91:4004-4008 (1994).

Owen C, Barnett M, Fitzgibbon J: Familial myelodysplasia and acute myeloid leukaemia - a review. Br J Haematol 140:123-132 (2008).

Panagopoulos I, Torkildsen S, Gorunova L, Ulvmoen A, Tierens A, et al: RUNX1 truncation resulting from a cryptic and novel $t(6 ; 21)$ (q25;q22) chromosome translocation in acute myeloid leukemia: a case report. Oncol Rep 36:2481-2488 (2016).
Ramsey H, Zhang DE, Richkind K, BurcogluO'Ral A, Hromas R: Fusion of AML1/Runx1 to copine VIII, a novel member of the copine family, in an aggressive acute myelogenous leukemia with $\mathrm{t}(12 ; 21)$ translocation. Leukemia 17:1665-1666 (2003).

Rodriguez-Perales S, Torres-Ruiz R, Suela J, Acquadro $\mathrm{F}$, Martin $\mathrm{MC}$, et al: Truncated RUNX1 protein generated by a novel $t(1 ; 21)$ (p32;q22) chromosomal translocation impairs the proliferation and differentiation of human hematopoietic progenitors. Oncogene 35:125-134 (2016).

Schuster C, Forster K, Dierks H, Elsasser A, Behre $\mathrm{G}$, et al: The effects of Bcr-Abl on C/EBP transcription-factor regulation and neutrophilic differentiation are reversed by the Abl kinase inhibitor imatinib mesylate. Blood 101:655663 (2003).

Sinclair PB, Sorour A, Martineau M, Harrison CJ, Mitchell WA, et al: A fluorescence in situ hybridization map of $6 \mathrm{q}$ deletions in acute lymphocytic leukemia: identification and analysis of a candidate tumor suppressor gene. Cancer Res 64:4089-4098 (2004).
Song X, Xia R, Li J, Long Z, Ren H, et al: Common and complex Notch1 mutations in Chinese oral squamous cell carcinoma. Clin Cancer Res 20:701-710 (2014).

Tsuzuki S, Hong D, Gupta R, Matsuo K, Seto M, Enver T: Isoform-specific potentiation of stem and progenitor cell engraftment by AML1/RUNX1. PLoS Med 4:e172 (2007).

Wang H, Chen X, Xu Z, Tan Y, Qi X, et al: Identification of a novel fusion gene, RUNX1$P R P F 38 A$, in acute myeloid leukemia. Int J Lab Hematol 39:e90-e93 (2017).

Wong TN, Ramsingh G, Young AL, Miller CA, Touma W, et al: Role of TP53 mutations in the origin and evolution of therapy-related acute myeloid leukaemia. Nature 518:552-555 (2015).

Yu YP, Ding Y, Chen Z, Liu S, Michalopoulos A, et al: Novel fusion transcripts associate with progressive prostate cancer. Am J Pathol 184: 2840-2849 (2014). 